\title{
A Characterization of Refinable Rational Functions
}

\author{
Paul Gustafson \\ Department of Mathematics \\ Texas A\&M University \\ College Station, Texas 77843-3368 USA \\ Nathan Savir \\ Department of Mathematics \\ Princeton University \\ Princeton New Jersey 08544-1000 USA
}

\author{
Ely Spears \\ Department of Mathematics \\ Rose-Hulman Institute of Technology \\ 5000 Wabash Avenue \\ Terre Haute, Indiana 47803 USA
}

Received:August 4, $2006 \quad$ Accepted: November 14, 2006

\begin{abstract}
In recent decades, refinable functions have become increasingly popular due to their desirable properties in many applications. Rational functions, however, are not as well-behaved as some other classes of functions and have seemingly escaped notice in terms of refinability. The authors spent the summer of 2006 investigating the refinability of rational functions while attending a National Science Foundation funded Research Experience for Undergraduates program at Texas A \& M University. Preliminary simplifications to the general case are presented in a chronological collection of lemmas. A complete characterization of refinable rational functions follows with an interesting connection to an open problem in number theory.

\section{INTRODUCTION}

The study of refinable functions has received a great deal of attention in the past few decades due to increased application in areas as diverse as computer graphics and signal processing. Classes of refinable functions, such as compactly supported splines and wavelet scaling functions, have been given special attention because they are easily extended for use in applications. Interested readers are encouraged to look into the results presented by Christiensen in [1]. Rational functions, however, are generally not continuous and are not necessarily square integrable. Undesirable traits such as these had caused the

refinability properties of rational functions to remain largely unnoticed. Here we present a characterization of refinable rational functions bearing striking connections to both the refinement characteristics of compactly supported splines and an open question in number theory.

\section{a. Preliminary Definitions}

Definition 1.1 A function $f: \mathrm{R} \rightarrow \mathrm{C}$ is said to be 2-refinable if there exists a sequence $\left(\mathrm{c}_{j \in Z}\right): c_{j} \in \mathrm{R}$ and

$$
f(x)=\sum_{j} c_{j} f(2 x-j) .
$$
\end{abstract}


If only finitely many of the terms in the sequence $\left(c_{j}\right)$ are non-zero, then the $f(x)$ is said to be finitely 2-refinable. For ease of notation, we will refer to such functions as refinable.

Definition 1.2 A function $f: \mathrm{R} \rightarrow \mathrm{C}$ is said to be almost refinable if there if there exists a sequence $\left(c_{j \in Z}\right): c_{j} \in R$ and

$$
f(x)=\sum_{i} c_{j} f(2 x-j)+K
$$

for some constant $\mathrm{K}$.

Definition 1.3 A function $f(x)$ is said to be a rational function if

$$
f(x)=\frac{P(x)}{Q(x)}
$$

where $P(x)$ and $Q(x)$ are polynomials in $x$ with no common factors and $Q(x)$ is not the zero polynomial.

Remark 1.4 Any rational function $f(x)$ can be rewritten via partial decomposition as the sum of a polynomial $G(X)$ and rational functions $H_{1}(x) \ldots H_{n}(x)$, where the rational functions $H_{i}(X)$ have the form

$$
H_{i}(x)=\frac{A}{(x-r)^{k}}
$$

or

$$
H_{i}(x)=\frac{B x+C}{\left(x^{2}+\alpha x+\beta\right)^{k}}
$$

with the denominator of this last form being irreducible and $A, B, C, r, k, \alpha$, and $\beta$ all being real constants.

The following section contains a few brief examples of refinable functions to help the reader form a better picture of refinability.

\section{b. Examples of Refinability}

Example 1.5 Let $f(x)=x^{2}+4 x+7$. Then

$$
\begin{aligned}
& f(2 x)=4 x^{2}+8 x+7 \\
& f(2 x-1)=4 x^{2}+4+4 \\
& f(2 x-2)=4 x^{2}+3
\end{aligned}
$$

and $f(x)$ satisfies the refinement given by

$$
\begin{aligned}
f(x)=\frac{21}{8} f(2 x)-\frac{17}{4} & f(2 x-1) \\
& +\frac{15}{8} f(2 x-2) .
\end{aligned}
$$

Thus, the sequence

$\left(c_{j}\right)=\left(\ldots, 0,0, \frac{21}{8}, \frac{-17}{4}, \frac{15}{8}, 0,0, \ldots\right)$, with only $\mathrm{c}_{0}, \mathrm{c}_{1}$, and $\mathrm{c}_{2}$ non-zero, is called the refinement sequence for $f(x)$.

Example 1.6 Let

$$
f(x)=\frac{-2}{x^{2}-1} .
$$

Using partial fraction decomposition, $f(x)$ can be rewritten as

$$
f(x)=\frac{-1}{x-1}+\frac{1}{x+1} .
$$

Then

$$
\begin{aligned}
& f(2 x-1)=\frac{-2}{4 x^{2}-4 x} \\
& f(2 x+1)=\frac{-2}{4 x^{2}+4 x}
\end{aligned}
$$

and $f(x)$ satisfies the refinement given by

$$
f(x)=2 f(2 x-1)+2 f(2 x+1) .
$$

As a closure to this section of examples, we present a proof that all polynomials of a single variable are refinable. We would like to point out that several students working with Dr. David Larson of Texas A \& M University during summer NSF-funded REU programs have come up with alternate proofs for this theorem. Though we could not find a paper explicitly containing the first proof of this theorem, Emily King was the first of Dr. Larson's students to present such a proof and did so during the summer of 2002 . The proof presented here is an original, obtained by the authors during the 2006 REU at Texas A \& M University.

Theorem 1.8 If $f(x)$ is a finite polynomial of a single variable, the $f(x)$ is finitely refinable.

Proof. Letting $\mathrm{n}$ be the degree of the polynomial, we may write 


$$
f(x)=\sum_{i=1}^{n} a_{i} x^{i}
$$

with $a_{n} \neq 0$ since the zero polynomial is trivially refinable. Substituting, we need to satisfy

$$
f(x)=\sum_{j \in Z i=0} \sum_{i}^{n} c_{j} a_{i}(k x-j)^{i} .
$$

By the binomial theorem, we get

$$
\begin{aligned}
f(x) & =\sum_{j \in Z, 0 \leq l \leq i \leq n} c_{j} a_{i}\left(\begin{array}{l}
i \\
l
\end{array}\right) k^{l} x^{l}(-j)^{i-l} \\
& =\sum_{l=0}^{n} x^{l} k^{l} \sum_{i=l}^{n} \sum_{j \in Z} a_{i}\left(\begin{array}{l}
i \\
l
\end{array}\right) c_{j}(-j)^{i-l}
\end{aligned}
$$

Comparing with the definition of $f(x)$ and setting coefficients equal gives us:

$$
k^{l} \sum_{i=l}^{n} \sum_{j \in Z} a_{i}\left(\begin{array}{l}
i \\
l
\end{array}\right) c_{j}(-j)^{i-l}=a_{l}
$$

for $0 \leq l \leq n$. Making the change of summation index $m=i-l$, the sum is rewritten as

$$
k^{l} \sum_{m=0}^{n-l} a_{m+l}\left(\begin{array}{c}
m+l \\
l
\end{array}\right) \sum_{j \in Z} c_{j}(-j)^{m}=a_{l}
$$

This is a triangular $n+1 \times n+1$ system of equations in the unknowns $\sum_{j \in Z} c_{j}(-j)^{m}$ with diagonal coefficients $a_{n} k^{l}$. Since $a_{n} \neq 0$ and $k \neq 0$, a solution exists. Now, pick any set of $n+1$ distinct integers, e.g. 1 to $n+1$, to be the set of $j$ 's with non-zero $c_{j}^{\prime}$ s. Then our problem of finding $c_{j}^{\prime}$ 's reduces to the matrix equation $A c$ $=\mathrm{b}$ where $\mathrm{A}$ is the $n+1 \times n+1$ matrix

$$
\left(\begin{array}{cccc}
1 & 1 & 1 & 1 \\
-j_{1} & -j_{2} & \ldots & -j_{n+1} \\
\left(-j_{1}\right)^{2} & \left(-j_{2}\right)^{2} & \ldots & \left(-j_{n+1}\right)^{2} \\
\vdots & \vdots & \ddots & \vdots \\
\left(-j_{1}\right)^{n} & \left(-j_{2}\right)^{n} & \ldots & \left(-j_{n+1}\right)^{n}
\end{array}\right)
$$

and $\mathrm{c}$ is the column matrix of the $c_{j}^{\prime} s$ and $\mathrm{b}$ is the column matrix of the $\sum_{j \in Z} c_{j}(-j)^{m}$ found above. But $\mathrm{A}$ is the transpose of a Vandermonde matrix with distinct row vectors, so its determinant is non-zero. Thus, we can compute $A^{-1} b$ to find the values of the $c_{j}$ 's.

\section{CONDITIONS FOR RATIONAL REFINABILITY}

The class of all rational functions is very large. Therefore, it is necessary to eliminate many rational functions with elementary properties that immediately prevent them from being refinable. After this simplification is finished, our sights can be set on finding conditions that are necessary and sufficient for rational functions of the remaining types to be refinable.

\section{a. Poles}

The term pole refers to a location where the denominator of a rational function vanishes. These locations can be along the real line or in the complex plane. Poles play the most important role in determining whether or not a rational function is refinable. In this section, we present several lemmas that whittle away the kinds of rational functions that cannot be refined due to properties of their poles.

Lemma 2.1 Let $f$ be a rational function. If $f$ has a complex pole, then it is not refinable.

Proof. Suppose $f$ has at least one complex pole. Assume for contradiction that $f$ is refinable. If this is the case, then both $f(x)$ and $\sum_{j} c_{j} f(2 x-j)$ have exactly the same poles. Let $h$ be the maximum of $\mathfrak{I}(z)$ over all poles $\mathrm{x}$ of $f$. Then all poles of $f(2 x-j)$ will be contained in the horizontal strip $|\mathfrak{I}(z)| \leq h / 2$. But $f$ has a pole $z_{0}$ such that $\left|\mathfrak{I}\left(z_{0}\right)\right|=h$, which is not contained in this strip. This is a contradiction and so $f$ is not refinable. 
Remark 2.2 Recall that in Remark 1.4, the standard forms for a rational function were discussed. Lemma 2.1 shows, after simplifying a rational function with partial fraction decomposition, that the denominator of each rational piece that remains (the functions $H_{i}(x)$ from Remark 1.4) must have only real roots. Therefore, any rational function of the form

$$
\frac{B x+C}{\left(x^{2}+\alpha x+\beta\right)^{k}}
$$

is never refinable unless the denominator has only real roots. If it does have only real roots, then it can be factored appropriately and partial fractions can be applied again until each piece of the rational function, $H_{i}(x)$, can be expressed in the form

$$
\frac{A}{(x-r)^{k}}
$$

where $k \in \mathbb{N}$ and $A$ and $r$ are real constants.

Lemma 2.3 Let $f$ be a rational function. If $f$ has a pole that does not occur at an integer, then $f$ is not refinable.

Proof. There are only three possible situations where that translates of poles of $\sum_{j} c_{j} f(2 x-j)$ can have a non-zero $c_{j}$.

1. The left-most pole of the contraction $f(2 x-j)$ occurs in the same location as the left-most pole of $f(x)$.

2. All the poles of the contraction mapping $f(2 x-j)$ occur at locations in between the left- and right-most poles of $f(x)$.

3. The right-most pole of the contraction mapping $f(2 x-j)$ occurs in the same location as the right-most pole of $f(x)$.

In other words, to include translates of $f(2 x)$ that have poles outside the region bounded by the left- and right-most poles of $f(x)$ would require infinitely many $c_{j}$ to ensure that $f(x)$ and $\sum_{j} c_{j} f(2 x-j)$ had exactly the same poles. From this we can assume without loss of generality that the left-most pole of $\sum_{j} c_{j} f(2 x-j)$ lines up with the left-most pole of $f(x)$, and any translate of $f(2 x)$ that shifts the poles to the left must have $c_{j}=0$.

Suppose $f$ has at least one noninteger pole. Then there is some pole $P$ such that $P$ is the left-most non-integer pole. Since $P$ is a non-integer, the mapping $f(2 x)$ will have a pole at $P / 2$, which is not an integer or half-integer (this pole at $P / 2$ will be the left-most pole in the function $f(2 x)$ that is not an integer or half-integer). Thus, no translation of poles occurring at integers can establish a pole at $x=P$ in the function $\sum_{j} c_{j} f(2 x-j)$. Since $P$ itself is non-integer, the distance between the points $P$ and $P / 2$ is not an integer or half-integer. Thus, translating a pole at $x=P / 2$ by integers will never result in a pole at $x=P$. The only other poles that can occur left of $x$ $=P$ are those that may fall in between $x=$ $P / 2$ and $x=P$. If one of these poles can be integer translated to line up with the pole at $x=P$, it must leave a new pole at the integer translate of the pole at $x=P / 2$. No translates of $f(2 x)$ can cover this new pole because it is the integer translate of the leftmost non-integer pole. Note also that there are no other non-integer poles to the left of $P$. We cannot left-translate the poles on the right side of $\mathrm{P}$ due the reasons given above. Therefore, there is no way to establish a pole at $x=P$ in the function $\sum_{j} c_{j} f(2 x-j)$ without introducing a new uncoverable pole at the translate of $x=P / 2$. Hence,

$$
f(x) \neq \sum_{j} c_{j} f(2 x-j)
$$

and $f$ is not refinable.

Lemma 2.4 Let $f(x)$ satisfy

$$
f(x)=\sum_{i \in Z} \frac{\alpha_{i}}{(x-i)^{k_{i}}}
$$

with at least one positive exponent $k_{i}$. If $\exists i, j \in Z$ such that $k_{i} \neq k_{j}$ then $f$ is not refinable. In other words, every pole of $f$ 
must be of the same order and $f$ must be a proper rational function.

Proof. Since $f$ has finitely many poles and at least one pole of positive order, it must have a left-most pole of positive order. Let this pole occur at $x=i$ and the order of the pole be $k_{i}>0$. Since $f$ has a pole of order $k_{i}$ at $x=i$, the mapping $\sum_{j} c_{j} f(2 x-j)$ must also have an order $k_{i}$ pole at $x=i$, if $f$ is to be refinable. The translation of poles that are not of order $k_{i}$ can ever establish an order $k_{i}$ pole. And from the same argument as in Lemma 2.3, we cannot use any pole occurring to the right of $x=i$ to establish a pole at $x=i$. This leaves only the option of using translates of the contracted version of the pole at $x=i$ itself to create a pole at $x=i$ in $\sum_{j} c_{j} f(2 x-j)$, requiring the equation

$$
c_{i} \frac{\alpha_{i}}{((2 x-i)-i)^{k_{i}}}=\frac{\alpha_{i}}{(x-i)^{k_{i}}} .
$$

Thus, $c_{i} \alpha_{i}=2^{k_{i}} \alpha_{i}$ and $c_{i}=2^{k_{i}}$. In order to establish a pole of order $n$, only translates of other poles of order $n$ can be used. This means that if $f(x)$ has poles of varying order, $f(x)$ can be separated into a sum of functions $f_{m}(x)$ where each $f_{m}(x)$ is a function that has poles all of the same order. Consequently, in order for $f(x)$ to be refinable, all of the functions $f_{m}(x)$ must be refinable with the same refinement sequence. However, as we noted a moment ago, the left-most pole of each order, $k_{n}$, will necessarily have a refinement coefficient of $c_{n}=2^{k_{n}}$. Thus, none of the functions $f_{m}(x)$ can have the same first refinement coefficient unless they have poles of the same order. Therefore, if $f(x)$ has poles of varying order. $f(x)$ is not refinable. Moreover, since $f(x)$ is assumed to have at least one pole of positive order, any refinable $f(x)$ cannot have a pole of negative order. This means that $f(x)$ cannot be reduced to the sum of a polynomial and a rational function, which implies that all rational refinable $f(x)$ must be proper rational functions.

In this section we have shown that for a rational function to be refinable, it can be reduced to a function $f$ that can be written in the following form:

$$
f(x)=\sum_{i \in Z} \frac{\alpha_{i}}{(x-i)^{k}},
$$

with every pole of $f$ having the same order. This is a rather strict preliminary simplification and so it may seem promising that a pattern will emerge fully characterizing the kinds of rational functions that are refinable. This idea is explored in the next section, leading up to a complete characterization of refinable rational functions.

\section{CHARACTERIZATION OF REFINABLE RATINAL FUNCTIONS}

After working out many examples of refinable rational functions, the authors noted that every refinable rational function seemed to have evenly spaced poles. That is, we conjectured that if some rational function $f(x)$ satisfying the conditions discussed in Section II is refinable, then $\exists N \in \mathrm{N}$ such that

$$
f(x)=\sum_{j} \frac{\alpha_{j}}{(x-(A+N j))^{k}}
$$

Where $A$ represents the left-most pole of $f(x)$. However, we uncovered some interesting counter-examples that exposed the need for more simplification before any meaningful insight would be attained.

\section{a. Counter-Examples}

Example 3.1 Let

$$
f(x)=\frac{1}{(x-4)}-\frac{1}{(x-1)}-\frac{1}{(x+1)}-\frac{1}{(x+4)} .
$$

Here the poles of $f(x)$ are not evenly spaced. Nonetheless, $f(x)$ is refinable with the refinement sequence 


$$
\begin{gathered}
f(x)=2 f(2 x-4)+2 f(2 x-1)+2 f(2 x+1) \\
+2 f(2 x+4)
\end{gathered}
$$

In fact, it can easily be verified (and the reader in encouraged to do so) that any rational function $f(x)$ of the form

$$
f(x)=\frac{1}{(x-A)}-\frac{1}{(x-B)}-\frac{1}{(x+B)}-\frac{1}{(x+A)}
$$

is refinable for $A, B \in Z^{+}: A>B$.

Remark 3.2 At this point, the perceptive reader may have noticed a few trends. In all of the examples of refinable rational functions, the only useful translates of the dilated original function are the translates corresponding to poles. Additionally, the issue of pole spacing still appears to be significant. Example 3.1 does not have evenly spaced poles, but it does have poles spaced symmetrically about the origin. Perhaps this is a strong enough condition to characterize all rational refinable functions.

Example 3.3 Let

$$
f(x)=\frac{1}{(x-5)}-\frac{1}{(x-2)}-\frac{1}{(x)}-\frac{1}{(x+3)} .
$$

Notice that this is the same function from Example 3.1, except that each pole has been translated one integer to the left. Now the poles are not evenly spaced and are not symmetric about the origin, yet $f(x)$ is still refinable (the refinement sequence from Example 3.1 still suffices if the translates are changed to match the poles of the new function).

Due to examples such as the previous two, some further simplifications are needed before proceeding to conjectures encompassing all refinable functions.

\section{b. Translations and Derivatives}

The previous example proves to be an important illustration of the fact that if all the poles of a rational function are translated by the same integer, the translation preserves refinability.

Lemma 3.4 Let $f(x)$ be refinable and have the form of equation (1). Then any function $g(x)$ such that

$$
g(x)=\sum_{i} \frac{\alpha_{i}}{(x-i+N)^{k}}
$$

for some fixed $N \in \mathrm{Z}$, is also refinable.

Proof. Choose $N \in Z$. Since $f(x)$ is refinable, we can write $f(x)$ and $g(x)$ as follows

$$
\begin{aligned}
& f(x)=\sum_{j} c_{j} f(2 x-j) \\
& g(x)=f(x+N)=\sum_{j} c_{j} f(2(x+N)-j)
\end{aligned}
$$

But $g(x)=f(x+N)$ and since $f(x+N)$ is refinable, then $g(x)$ is refinable.

Lemma 3.5 Let $f$ be a proper rational function. Then $f$ is refinable if and only if the derivative $f^{\prime}$ is refinable.

Proof. ( $\Rightarrow$ ) Let $f$ be a refinable function. Then $f$ satisfies equation (2). Taking the derivative of each side yields

$$
\begin{aligned}
f^{\prime}(x) & =2 \sum_{j} c_{j} f^{\prime}(2 x-j) \\
& =\sum_{j} d_{j} f^{\prime}(2 x-j)
\end{aligned}
$$

where the refinement sequence $\left(d_{j}\right)$ is just $\left(2 c_{j}\right)$.

$(\Leftarrow) \quad$ Let $f^{\prime}$ be refinable. Then $f^{\prime}(x)=\sum_{j} c_{j} f^{\prime}(2 x-j)$. Thus, $f$ and the function $g$, where $g(x)=\sum_{j} \frac{c_{j}}{2} f(2 x-j)$ are two rational functions with the same derivative. This implies that their difference is a constant, i.e.,

$$
f(x)=\sum_{j} \frac{C_{j}}{2} f(2 x-j)+K
$$

for some constant $K$. Taking the limit of each side of this equation as $x \rightarrow \infty$, we see that since $f$ and $g$ are proper rational functions, $\lim _{x \rightarrow \infty} f(x)=0$ and $\lim _{x \rightarrow \infty}$ $g(x)=0$. Therefore the constant $K$ must be zero, meaning $f=g$, and $f$ is refinable with refinement sequence $\frac{c_{j}}{2}$. 
With these lemmas in mind, we can make two important simplifications to the problem of characterizing refinable functions. First note that differentiating a rational function of the form of equation (1) will just yield another rational function of the same form with poles of a higher order. Therefore, only rational functions with poles of first order need to be considered. Second, we are free to translate the poles of a given function by any integer without affecting refinability. So, without loss of generality we can assume the left-most pole of all the functions $f(x)$ considered occurs at $x=0$. With these two simplifications in mind, we can now develop necessary and sufficient conditions for the refinability of rational functions.

\section{c. Scaling Polynomials and Divisibility}

From this point forward, we shall assume that all rational functions under consideration for refinability have the following form

$$
f(x) \sum_{i=0}^{N} \frac{\alpha_{i}}{(x-i)}
$$

$\alpha_{i} \in \mathrm{R}$. Since we are not considering every possible pole between $i=0$ and $i=\mathrm{N}$, some of the $\alpha_{i}$ may be zero. The cases of $i=0$ and $i=\mathrm{N}$, however, can be assumed to have non-zero $\alpha$ values.

In order for some rational function $f(x)$ to satisfy equation (2), the translates of the contraction mapping $f(2 x)$ have to be lined up onto the original poles found in $f(x)$. In the contraction $f(2 x)$, the distance of each pole of $f(x)$ from the origin will be halved. For example, the function

$$
f(x)=\frac{1}{x-1}-\frac{1}{x-2}+\frac{1}{x-4}
$$

has poles at $x=1, x=2$, and $x=4$. The contraction mapping $f(2 x)$ will have poles at $x=1 / 2, x=1$, and $x=2$. If $f(x)$ is to be refinable, then the translates of $f(2 x)$ must be used to eliminate the pole at $x=1 / 2$ and create a pole at $x=4$. To illustrate this point, let us reconsider Example 3.1. By expanding the terms in the refinement of $f(x)$, the cancellation of poles becomes more apparent.

$$
\begin{aligned}
& f(2 x-4)=\frac{1}{(2 x-8)}-\frac{1}{(2 x-5)}-\frac{1}{(2 x-3)}+\frac{1}{(2 x)} \\
& f(2 x-1)=\frac{1}{(2 x-5)}-\frac{1}{(2 x-2)}-\frac{1}{(2 x)}+\frac{1}{(2 x+3)} \\
& f(2 x+1)=\frac{1}{(2 x-3)}-\frac{1}{(2 x)}-\frac{1}{(2 x+2)}+\frac{1}{(2 x+5)} \\
& f(2 x+4)=\frac{1}{(2 x)}-\frac{1}{(2 x+3)}-\frac{1}{(2 x+5)}+\frac{1}{(2 x+8)}
\end{aligned}
$$

Notice how each equation conveniently provides terms that cancel out unwanted poles in some of the other equations. When the dust settles, only those terms that have denominators found somewhere in the original function are left. The first term of equation (4) illustrates this well. The denominator is $2 x-8=2(x-4)$. Thus, this term contributes the necessary pole at $x=4$, but with a new coefficient (scaled by $1 / 2$ ) in the numerator. From conditions like this, one can write a system of equations that uniquely determines the refinement sequence $\left(c_{j}\right)$ and implies the refinability of the function $f$.

To emphasize this point just a little more, consider the translates of some contraction mapping $f(2 x)$. The constants $c_{j}$ reveal how far we have translated from $x$ 
$=0$. So if $j=3$, it means we have translated the poles of $f(2 x)$ by three half-integers. The constants $\alpha_{i}$ reveal which pole we are considering. So if $i=2$, it means we're located at $x=1$ (the pole that is half the distance from the original pole located at $x=$ 2 in the function $f(x))$. Thus, the term $\mathrm{c}_{3} \mathrm{a}_{2}$ corresponds to the "weight" of the pole at $x$ $=2.5$ contributed by the pole that was originally at $x=2$ in $f(x)$. The term $c_{1} \alpha_{4}$ will also contribute some "weight" to the pole at $x=2.5$. This is because the $1^{\text {st }}$ translate (one half-integer) of the contraction of the pole at $x=4$ (so really this is $x=2$ in the $f(2 x)$ world) will occur at $x=2.5$. The sum of all of these terms which contribute weight to a given pole must be the same as the original weight from $f(x)$, up to a scale factor introduced due to the contraction. This allows us to write an equation associated with each half-integer from $x=0$ to $x=N$. The equations will represent whether or not there was a pole in the original function $f(x)$ and what the numerator of the pole was in $f(x)$. If the equations are satisfied, then there will exist a sequence of refinement constants such that $f(x)$ satisfies equation (2).

$$
\begin{aligned}
& \text { For } f(x) \text { to satisfy } \\
& f(x)=\sum_{i=0}^{N} \frac{\alpha_{i}}{(x-i)}=\sum_{j=0}^{N} c_{j} f(2 x-j)
\end{aligned}
$$

the following equations are sufficient and necessary:

$$
\begin{aligned}
2 \alpha_{0} & =c_{0} \alpha_{0} \\
0 & =c_{1} \alpha_{0}+c_{0} \alpha_{1} \\
2 \alpha_{1} & =c_{2} \alpha_{0}+c_{1} \alpha_{1}+c_{0} \alpha_{2} \\
\vdots & \vdots
\end{aligned}
$$

$$
\begin{gathered}
0=c_{N} \alpha_{N-1}+c_{N-1} \alpha_{N} \\
2 \alpha_{N}=c_{N} \alpha_{N}
\end{gathered}
$$

Careful inspection of these equations reveals that the sequence of solutions

$$
\left(2 \alpha_{0}, 0,2 \alpha_{1}, \ldots, 0,2 \alpha_{N}\right)
$$

is the convolution of the two sequences $\left(c_{j}\right)$ and $\left(\alpha_{j}\right)$. We employ the z-transform of each sequence in order to rewrite this as a polynomial multiplication.

Definition 3.6 For a function

$$
f(x)=\sum_{i=0}^{N} \frac{\alpha_{i}}{(x-i)}
$$

the scaling polynomial of $f$ is the polynomial $S(z)$ such that

$$
\begin{aligned}
S(z) & =\alpha_{0}+\alpha_{1} z+\alpha_{2} z^{2}+\ldots+\alpha_{N} z^{N} \\
& =\sum_{i=0}^{N} \alpha_{i} z^{i}
\end{aligned}
$$

The refinement polynomial of $f(x)$ is the polynomial $R(z)$ such that

$$
\begin{aligned}
R(z) & =c_{0}+c_{1} z+c_{2} z^{2}+\ldots+c_{N} z^{N} \\
& =\sum_{j=0}^{N} c_{j} z^{j}
\end{aligned}
$$

From these definitions we can see that the convolution of the sequences $\left(c_{j}\right)$ and $\left(\alpha_{j}\right)$ is just the sequence of coefficients in the product of $S(z)$ and $R(z)$. When we write the polynomial generated by the solution sequence, we must have

$$
\begin{aligned}
S(z) R(z) & =2 \alpha_{0}+0 z+2 \alpha_{1} z^{2}+0 z^{3}+\ldots+2 \alpha_{N} z^{2 N} \\
& =2\left(\alpha_{0}+0 z+\alpha_{1} z^{2}+0 z^{3}+\ldots+\alpha_{N} z^{2 N}\right) \\
& =2 S\left(z^{2}\right)
\end{aligned}
$$

Theorem 3.7 A rational function

$$
f(x)=\sum_{i=0}^{N} \frac{\alpha_{i}}{(x-i)} \text { is refinable if and only if }
$$

the scaling polynomial, $S(z)$, satisfies $S(z) \mid S\left(z^{2}\right)$. 
Proof. $(\Rightarrow)$ Let $f(x)=\sum_{i=0}^{N} \frac{\alpha_{i}}{(x-i)}$ be refinable. Then there exist sequences $\left(c_{j}\right)$ and $\left(\alpha_{j}\right)$ such that equation (5) through (9) are satisfied. By constructing polynomials

$$
R(z)=\sum_{i=0}^{N} \alpha_{i} z^{i} \text { and } S(z)=\sum_{j=0}^{N} c_{j} z^{j},
$$

we have the existence of a scaling polynomial and refinement polynomial such that $S(z) R(z)=2 S\left(z^{2}\right)$ and therefore $S(z) \mid S\left(z^{2}\right)$.

$(\Leftarrow)$ Suppose that the scaling polynomial $S(z)$ of some rational function $f$ satisfies $S(z) \mid S\left(z^{2}\right)$. Then define

$$
R(x)=\frac{2 S\left(z^{2}\right)}{S(z)}
$$

And since $S(z) R(z)=2 S\left(z^{2}\right)$ we know that the sequence of coefficients of terms in $S(z)$ along with the corresponding sequence from $R(\mathrm{Z})$ will satisfy equations (5) through (9). Thus, $f$ is refinable and its refinement sequence is given by the coefficients of the polynomial

$$
\frac{2 S\left(z^{2}\right)}{S(z)}
$$

\section{CONCLUSIONS}

From the result in Theorem 3,7, the problem of characterizing refinable rational functions can be reduced to finding polynomials $S(z)$ such that $S(z) \mid S\left(z^{2}\right)$. This allows us to place a restriction on the kinds of roots the polynomial $S(z)$ can have. Make sure not to confuse the roots of this polynomial with the poles of the rational function used to create the polynomial.

\section{a. Characterizing the Roots of $S(z)$}

Lemma 4.1 Let the polynomial $S(z)$ satisfy $S(z) \mid S\left(z^{2}\right)$. If $r$ is a root of $S(z)$ then either $r=0$ or $|r|=1$.

Proof. Since $r$ is a root of $S(z)$ the quantity $(x-r)$ divides $S(z)$ and so $r$ is also a root of
$S\left(z^{2}\right)$. But if $r$ is a root of $S\left(z^{2}\right)$, then $r^{2}$ must have been a root $S(z)$. If $r$ is a root of $S(z)$, then there exists a sequence $\left(r_{0}, r_{1}, r_{2}, \ldots\right)$ such that

$$
\begin{aligned}
& r_{0}=r \\
& \left|r_{n}\right|=\sqrt{\left|r_{n-1}\right|}
\end{aligned}
$$

and $r_{n}$ is a root of $S(z)$ for each $n \in \mathbb{N}$. If $|r| \notin\{0,1\}$ then $r_{n}=r_{m} \Rightarrow n=m$, which means that $S(z)$ has infinitely many roots. Therefore, all roots $r$ of $S(z)$ must have $r=0$ or $|r|=1$. Note that if we consider that $S(z)$ to be a scaling polynomial for some rational function, the constant $\alpha_{0}$ will be non-zero, so the case $r=0$ is irrelevant for scaling polynomials.

From the above restriction, we must have that the sequence $\left(r_{n}\right)$ of roots of $S(z)$ eventually cycles, meaning that for sufficiently large $i$ there exists $N$ such that

$$
\left(r_{i}\right)=\left(r_{i}\right)^{2^{N}} \text {. }
$$

In other words, each $r_{i}$ is an integer root of unity. So suppose that

$$
r_{i}=e^{\frac{2 \pi i j}{k}}
$$

where $k$ divides $2^{N}-1$. If this is the case, then the cycle of roots is determined by the multiplicative group generated by 2 in $\mathrm{Z} / \mathrm{kZ}$. Characterizing this group for general $k$ is an open problem in number theory. Interested readers are encouraged to look into [2].

\section{b. Generalizing to Spline Functions}

The poles of a rational function represent the places where such a function is not analytic. From this, it is a reasonable conjecture that a similar approach to the refinability of any class of functions which can be characterized by points of nonanalytic behavior will work. As it turns out, such an approach was undertaken by Lawton, Lee, and Shen in [3]. Their work shows that a similar result to the one obtained for rational functions will also characterize the refinability conditions for compactly supported splines. This result is highly applicable to wavelet theory because of the usefulness of splines in approximation. The natural progression from these single-variable spline functions is 
the study of multivariate functions. This motivated further investigation of the refinability of splines of two variables.

c. Generalizing to Two or More Variables

Applying the same approach as above, we found that the problem of refinable multivariable splines (or even rational functions) can be restated more clearly using algebraic geometry. Let $A \subset C^{N}$ be the algebraic variety of some polynomial $\mathrm{P}\left(x_{1}, \ldots, x_{n}\right)$-that is,

$$
A=\left\{\left(y_{1}, \ldots, y_{n}\right) \mid P\left(y_{1}, \ldots, y_{n}\right)=0\right\} .
$$

Then a function that is not analytic on $A$ can be refinable if and only if $A=\cup_{j}\left(2^{-1} A+j\right)$. We conjecture that if $A$ satisfies this condition, then $A$ is a union of complex lines.

\section{ACKNOWLEDGEMENTS}

We would like to thank David Larson and Leonid Kovalev of Texas A \& M University for their helpful advice and invaluable mentoring. The National Science Foundation also deserves special recognition for funding our research experience. We would also like to thank Wayne Lawton for his help in obtaining the reference about the refinability of splines. Finally, we would like to recognize on of our fellow researchers, Joel Gomez, for his help in explaining some of the basics of algebraic geometry.

\section{REFERENCES}

1. Ole Christensen and Khadija Laghrida Christensen, "Linear Independence and Series Expansions in function Spaces," Amer. Math. Monthly, Vol. 113, No. 7, pages 611-627, 2006.

2. G. H. Hardy and E. M. Wright, An Introduction to the Theory of Numbers (Fifth Edition, Clarendon Press, Oxford, 1979) chapter 6.

3. Wayne Lawton, S.L. Lee, and Z. Shen, "Complete characterization of refinable splines," Advances in Computational Mathematics, Vol. 3, pages 137-145, 1995.

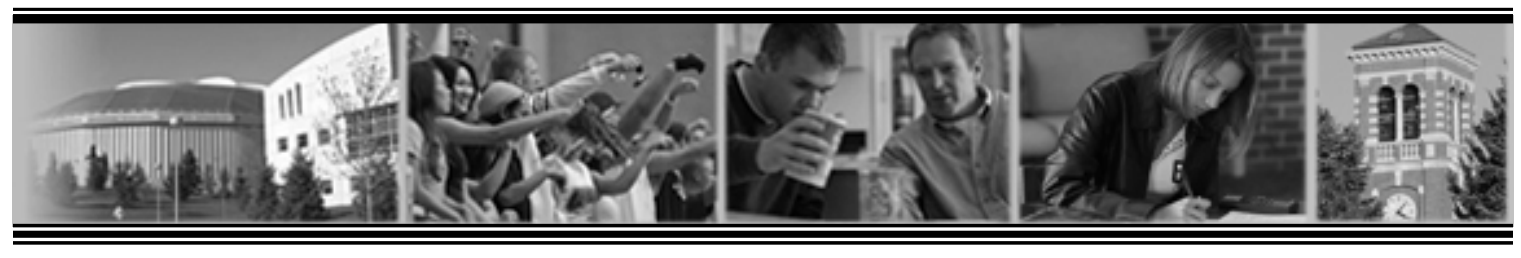

\section{University of Northern lowa: Cedar Falls, lowa USA}
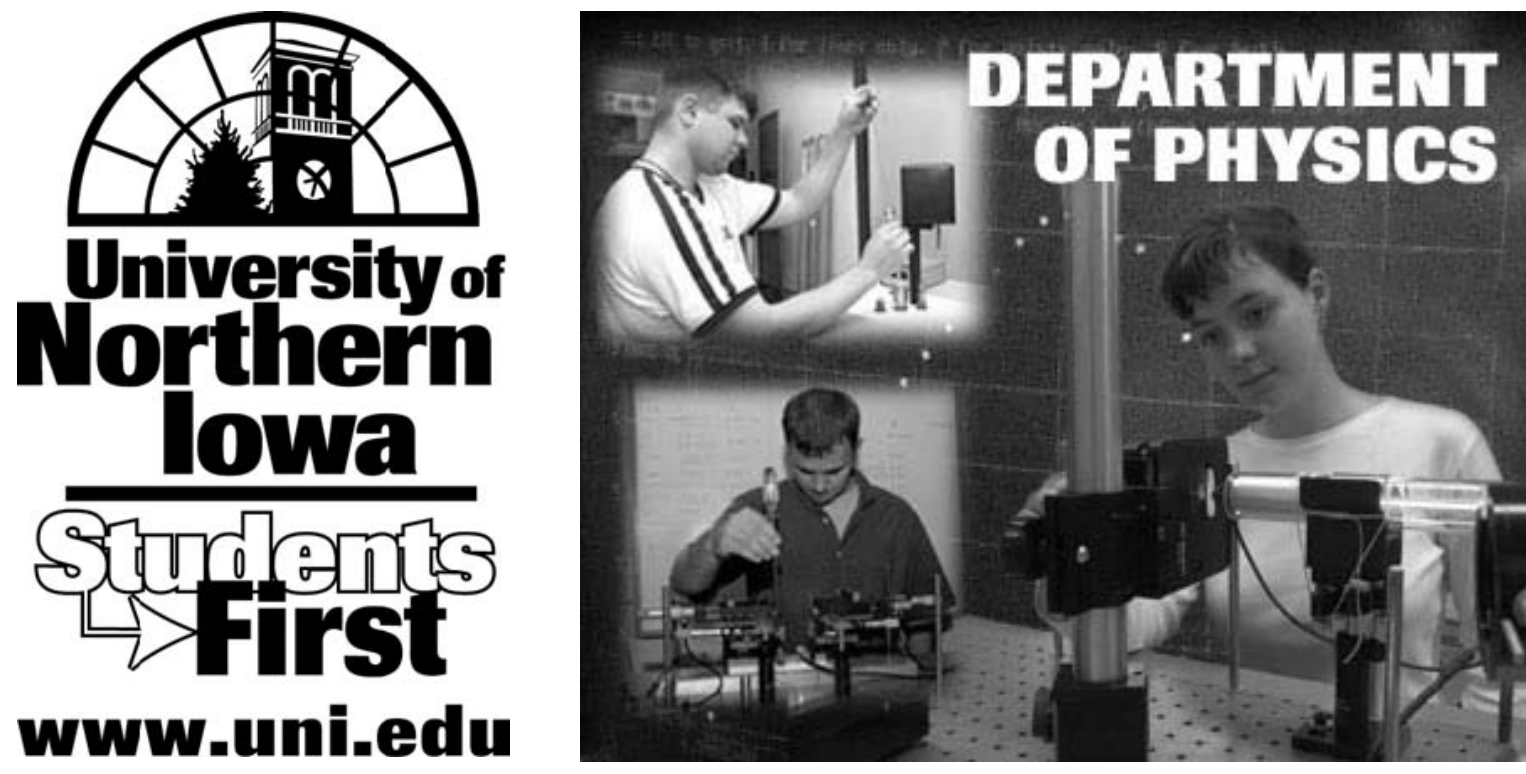